livraisons

d'Histoire

de l'Architecture

\section{Livraisons de l'histoire de l'architecture}

42 | 2021

2001-2021 / numéro anniversaire

\title{
Droit et architecture, discours autour de la figue de l'architecte auteur
}

Law and architecture, a discourse on the figure of the architect as author

Recht und Architektur, ein Diskurs über die Figur des Autorenarchitekten

\section{Marie Cornu}

\section{(2) OpenEdition}

Journals

Édition électronique

URL : https://journals.openedition.org//ha/4050

DOI : $10.4000 /$ /ha. 4050

ISSN : 1960-5994

Éditeur

Association Livraisons d'histoire de l'architecture - LHA

Référence électronique

Marie Cornu, «Droit et architecture, discours autour de la figue de l'architecte auteur », Livraisons de I'histoire de l'architecture [En ligne], 42 | 2021, mis en ligne le 11 décembre 2021, consulté le 11 décembre 2021. URL : http://journals.openedition.org//ha/4050 ; DOI : https://doi.org/10.4000//ha. 4050

Ce document a été généré automatiquement le 11 décembre 2021.

Tous droits réservés à l'Association LHA 


\section{Droit et architecture, discours autour de la figue de l'architecte auteur}

Law and architecture, a discourse on the figure of the architect as author Recht und Architektur, ein Diskurs über die Figur des Autorenarchitekten

\section{Marie Cornu}

1 Le thème de l'architecture comme processus de création, présent dans de nombreuses Livraisons d'histoire de l'architecture ne manque pas de poser la question qu'entretiennent le droit et l'architecture.

2 Ces rapports sont multiples : l'architecture exprimant une certaine idée du droit, de la justice et de la façon dont elle est administrée (les bâtiments judiciaires par exemple); le droit lui-même vu comme une construction, les métaphores sont nombreuses et suggestives: pour n'en prendre qu'une et des plus fameuses, la pyramide de Kelsen figurant la hiérarchie des normes; le droit et l'architecture pris comme deux façons jumelles d'ordonner l'espace ${ }^{1}-$ ils ont des affinités culturelles ${ }^{2}-$ l'architecture dans ses liens étroits avec le droit, elle est un art de faire avec la contrainte. Elle est pétrie de règles. Le droit est partout inscrit dans la pierre (hauteur du bâti, distance d'avec l'autre, servitudes de vue, accès au public, etc.). Les deux sont aussi, selon leurs modes opératoires un certain art de relier l'abstraction et la réalité. Il y a selon Robert Carvais et Dirk Van de Vijver, « une analogie structurale plus décisive entre ces deux savoirs, un geste scientifique commun et double, une réflexion théorique et une application pratique $»^{3}$. Les trois principes de Vitruve (Firmitas - la solidité - Utilitas - l'usage, la fonction - Venustas - la beauté) ne sont-ils pas posés «à la manière du droit romain [...] en quelque sorte suspendus hors du temps et de l'espace ${ }^{4}$ »?

3 C'est le rapport de leurs proximités et leurs distances que j'ai choisi d'explorer ici dans un espace particulier, celui de la perception de l'architecture/œuvre. Il ne s'agit pas de chercher à mettre en lumière, dans les travaux savants publiés dans LHA, une réflexion ou une référence au droit ou encore d'extraire ce qui pourrait ressembler à un appel au 
droit. L'histoire du droit d'auteur est émaillée d'affaires qui ont concerné des réalisations architecturales et nous en évoquerons certaines. Mais l'idée ici est plutôt de questionner les espaces de rencontre entre ces deux sciences, de comprendre en quoi les revendications sociales croisent ou non de possibles revendications juridiques. Quel écho renvoie dans le champ du droit la réflexion sur l'architecture? Que peut nous enseigner ce côtoiement de la règle et de l'art et à quel moment le récit de l'architecture croise, alimente, enrichit celui du droit? Dans cet exercice, je me concentre sur la question très présente dans la revue LHA, de l'architecture comme processus de création et dans cette veine, de celle de la figure de l'architecte-auteur. C'est en l'occurrence une entrée des plus visibles dans la revue, un des points centraux de contact avec le droit, ce que disent les vocabulaires que mobilisent historiens, historiens d'art, conservateurs quand ils parlent d'architecture. Droit et architecture ont assurément un vocabulaire partagé. On peut, partant de ces modes de nomination, se demander jusqu'à quel point leur façon de saisir l'œuvre, de découper le réel se rejoignent.

\section{L'architecture/œuvre, retour vers l'histoire du droit}

Comment s'y prend le droit pour saisir la création, pour en faire une catégorie du droit ? Il semble agir au plus près de la réalité lorsqu'il décide que l'auteur a des droits du seul fait de la création. C'est le premier article du Code de la propriété intellectuelle. C'est l'acte de créer, en droit français, qui institue l'auteur et partant, qui fait qu'il est propriétaire de son œuvre. Il n'y ici aucune place pour la fiction, technique pourtant familière au légiste. Les personnes morales ne sont pas des auteurs en droit français. Mais la question reste entière de savoir ce qu'est ce fait de création. Les textes révolutionnaires inaugurant une nouvelle façon de penser le droit d'auteur énuméraient ceux qui devaient bénéficier d'une protection : «les auteurs d'écrits en tout genre, les compositeurs de musique, les peintres et dessinateurs qui feront graver des tableaux ou dessins ${ }^{5}$ ", et donnaient quelques exemples à titre illustratif. Le juge ne s'en tiendra pas à cette liste, il y verra plus largement le besoin de protéger les beauxarts et belles lettres contre les pirates. À ce moment de l'histoire du droit, il y a comme une évidence quant au cercle de protection ${ }^{6}$. Ce sont les productions du génie humain ${ }^{7}$ qui sont ici visées et on sait ce qu'est cette réalité des arts et lettres. Le droit, n'est d'aucun recours dans leur qualification. Il saisit ce fait de la création, tient pour acquis qu'il puisse s'énoncer dans une autre sphère que celle du droit. Le besoin d'une frontière tracée de l'intérieur, posée par la règle, viendra plus tard lorsque précisément apparaissent de nouvelles formes jusque-là inconnues, de nouvelles productions pour certaines issues de la société industrielle: les arts dits mécaniques, les multiples (les statuettes proposées au commerce ou les éléments décoratifs produits en série). Le besoin d'une frontière par l'interposition d'une notion juridique d'œuvre, forgée par la jurisprudence du second XIXe, vient de ce que la partition entre ce qui relève de la création et ce qui devrait en être exclu n'est plus si évidente. En particulier, certains couples d'opposition se révèlent : celui, en forte tension de l'art et de la technique, le second pourrait-il disqualifier le premier ? C'est aussi dans ces périodes que l'on discute de la partition entre le beau et l'utile. Pouillet exposant l'état des doctrines et de la jurisprudence en grande partie réticente à reconnaître l'art dans des objets de commerce, plaide au contraire l'indifférence de la destination de l'œuvre. Elle ne " change pas le caractère du droit ${ }^{8}$. Étonnamment, la question de la protection des 
œuvres d'architecture ne s'inscrit pas dans ce débat ${ }^{9}$. Du moins Pouillet aborde-t-il la question distinctement: "L'importance et la nouveauté de la question du droit des architectes, en ce qui touche les œuvres des architectes nous ont naturellement conduit à la traiter à part $»^{10}$. Prenant à rebours les arguments hostiles à la reconnaissance de l'architecte auteur, il estime au contraire qu'en dépit du silence de la loi - elle ne parle pas des architectes ${ }^{11}$ - « en parlant de l'art du dessin, ne parle-t-elle pas implicitement et presque nécessairement de l'architecture, qui ne vit que de lignes, c'est-à-dire du dessin $»^{12}$. Où l'on aperçoit ici l'importance de cette entrée par le dessin de l'architecture. Il est «la pointe visible du travail architectural» dans la pensée de Viollet-le-Duc ${ }^{13}$. Le dessin d'architecture " possède en soi une valeur d'art (qui) range nécessairement l'architecte, aux yeux même de ceux qui en douteraient, dans la catégorie des plasticiens, des artistes, des créateurs $»^{14}$. L'architecte auteur, en réalité, est depuis longtemps, une figure reconnue ${ }^{15}$. La Cour d'appel de Paris décide en 1855 "qu'en raison de l'élévation de la pensée qui a présidé à leur conception et du mérite de leur exécution, les œuvres d'architectures doivent être considérées comme des œuvres d'art $\aleph^{16}$. Ce qui est en débat dans ces périodes de la fin du XIX dans la profession notamment ${ }^{17}$, n'est pas tant la question de la dimension artistique des œuvres architecturales - personne n'en doute - que celle de l'opportunité d'allouer des droits, plus spécialement de la possibilité de réserver cet espace, d'en privatiser une partie. Et cette discussion signale le rapport singulier qu'entretient ce champ de la création à l'espace public. S'exprime ici, encore confusément, l'idée que l'architecture obéit à des règles propres. Nous y reviendrons. Quoi qu'il en soit, la question de la qualification d'œuvre de l'esprit à propos de l'œuvre architecturale ne manque pas de se poser.

\section{Idée, forme, originalité, les épreuves de qualification}

5 Le droit, dans son entreprise de délimitation de l'œuvre, tente d'incertaines frontières entre les idées et les formes. On protège la seconde si elle est originale, non la première, du moins en principe. Plus justement, ce n'est que prise non pas dans la forme mais en tant que forme qu'elle reçoit une protection juridique. Au dehors, chacun peut y puiser à sa guise : «quelle qu'en soit l'ingéniosité et même si elles sont marquées au coin du génie, la propagation et l'exploitation des idées exprimées par autrui ne peut être contrariée par les servitudes inhérentes au droit des auteurs : elles sont par essence et par destination de libre parcours $»^{18}$. Seule $"$ la forme d'expression et non l'idée exprimée » est susceptible d'appropriation. C'est la forme et non le fond que voit le droit. Improbable (parfois impossible) partition. En architecture, la question des formes est sans aucun doute un sujet, mais elle semble être avant tout pensée dans sa liaison intime avec l'idée qui les porte ${ }^{19}$, ce que suggère cette belle image de « la pensée-dessin de J.-P. Jungmann (comme) émanation du trinôme œil/main/papier ${ }^{20}$. Le dessin d'architecture «se distingue [...] par la relation que la forme entretient avec l'idée : davantage que son homologue en peinture et en sculpture, il s'implique dans un dialogue avec un tiers auquel il doit communiquer les différentes phases dans l'élaboration du projet [...] Plus que tout autre, le dessin d'architecture donne la trace du travail de conception qui s'effectue depuis la cosa mentale jusqu'à l'immeuble

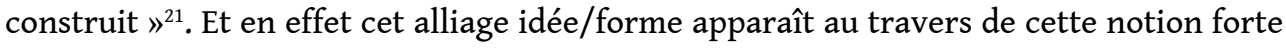
de projet $^{22}$. 
6 Vu du droit, le critère de la forme est partout dans l'art de bâtir : le dessin d'abord, dans ses différents états d'achèvement (y compris esquisses, brouillons, ébauches), en avantposte dans le champ du droit de la création tout autant que la maquette d'architecture qui peut «avoir une existence artistique ou conceptuelle propre, [...] relativement indépendante du projet qu'elles (représentent) ${ }^{23}$ ", toutes traces qui documentent le travail de l'architecte et bien sûr le bâtiment construit. Mais au-delà de la valeur documentaire que peuvent avoir certains de ces jalons, chacun peut être vu comme une œuvre. Le droit ne prend guère en compte les liens qui les relient. Ces multiples mises en forme de la pensée architecturale sont sans aucun doute de celles qui donnent prise à des droits d'auteurs, même si on a éprouvé le besoin de préciser dans la loi fondatrice de 1957 le statut des plans-type. Le plan lui-même eût trouvé abri sous la catégorie des dessins mais il n'était pas sûr que le soit l'exécution matérielle de ce plan en forme de projection d'une chose à construire: "le Parlement a entendu soumettre au consentement de l'architecte distinctement l'édification de chacun des bâtiments, qui sont construits à partir d'un plan-type ", mise au point rendue nécessaire au regard des pratiques abusives émanant notamment de l'administration ${ }^{24}$.

7 C'est sans doute sur le terrain de l'originalité, autre invention du droit pour qualifier l'œuvre, que l'épreuve est la plus sérieuse pour l'architecture, tout à la fois art et technique, assise sur ses trois piliers de l'utilitas, la firmitas, la venustas. «C'est la conjugaison active des trois qui est pertinente. Les trois notions sont indépendantes, sans hiérarchie, toute construction devant les conjuguer toutes trois sans en négliger aucune. Pour autant elles ne sont pas nécessairement étanches : il peut y avoir du venustas dans firmitas ou dans utilitas ou inversement du firmitas dans venustas etc. ${ }^{25}$. Et le juge ne saisit pas toujours cette combinatoire, ne voit pas que les uns ne vont pas sans les autres. Il refuse par exemple protection à des réalisations dont il estime qu'elles relèvent non de l'art mais de la technique et qu'ainsi elles ne présentent " pas un caractère suffisamment original pour invoquer des droits, en particulier pour que l'architecte exige " que son nom fût inscrit sur la façade de la préfecture ${ }^{26}$. Si l'on peut admettre que certaines réalisations ne font que reproduire des formes ou des idées qui sont déjà là, qu'il y aurait des constructions banales quand d'autres sont originales ${ }^{27}$, la motivation des décisions, le plus souvent montre l'embarras du juge dans l'appréhension de l'architecture comme art. L'exemple de la Maison Girard conçue par M. Sirvin est édifiant de ce point de vue. Les juges citent les revues d'architectures nationales ou étrangères qui la reconnaissent « sous des qualificatifs divers comme une création originale ", mais ils ne manquent pas de manifester leur incompréhension de l'œuvre. En forme d'antiphrase, ils admettent que l'originalité de la maison «ne prête pas sérieusement à discussion, même si elle pouvait échapper aux profanes, s'agissant d'une sorte de cube de béton, planté sur des poteaux de béton... $»^{28}$. Évoquant la société propriétaire du terrain qui l'avait détruite, les juges disent encore que son attention n'avait «à aucun moment été attirée sur l'originalité de l'ouvrage litigieux, laquelle ne tombe pas à la vérité sous le sens ». L'œuvre est originale, soit, mais sa destruction ne sera pas considérée comme attentatoire aux droits de son auteur. Nous avons jusqu'ici parlé de l'auteur mais la création architecturale peut aussi s'inscrire dans une logique collective, être le produit de plusieurs auteurs, de plusieurs contributeurs qui, sans prétendre à la qualité d'auteur, sont parties prenantes de la création et le droit ne les voit pas $^{29}$. La propriété littéraire et artistique inscrit son histoire avant tout dans un rapport individuel auteur-œuvre, même si des figures de propriété collective existent ${ }^{30}$ et l'histoire de l'architecture semble bien être une histoire par auteurs [...], c'est-à-dire 
par architectes et par architectures suivant un modèle standard anhistorique et antihistorique $»^{31}$.

Une autre des singularités de l'œuvre architecturale réside dans le fait qu'elle est foncièrement un lieu de partage d'utilités.

\section{L'architecture, lieu de partage d'utilités}

9 Si l'architecture est un espace de droit privatif pour son auteur, il se trouve borné par d'autres usages, par d'autres utilités privées et publiques. Il affronte par exemple le propriétaire du corpus de son œuvre. Clarifiant une jurisprudence peu stabilisée sur le sujet, le législateur a depuis le début du siècle posé cette cloison entre propriété matérielle et propriété immatérielle. La première n'emporte pas la deuxième du moins par le seul effet de la loi. Les deux sont dites indépendantes, chaque titulaire, de son côté, est censé exercer son droit. La partition n'évite pourtant pas que ces usages distincts de l'œuvre puissent entrer en conflit et le droit doit trancher. C'est précisément à propos de l'œuvre architecturale que s'est élaborée toute la jurisprudence sur les œuvres dites à caractère utilitaire, inaugurée par l'arrêt Bull lequel crée une sorte de protection à deux vitesses avec d'un côté les œuvres qui ont un usage, une fonction et de l'autre celles qui relèvent de l'art pour l'art ${ }^{32}$. L'architecture est « rêve et fonction ». L'architecte compose, en amont, avec ce donné. Mais une fois l'œuvre achevée et livrée, il n'est pas à l'abri de modifications ultérieures dictées par l'usage. Le juge en reconnaît la légitimité sous certaines conditions. Reprenant la formule des juges d'appel, le juge de cassation consolide le principe selon lequel «la vocation utilitaire du bâtiment commandé à un architecte interdit à celui-ci de prétendre imposer une intangibilité absolue de son œuvre, à laquelle son propriétaire est en droit d'apporter des modifications lorsque se révèle la nécessité de l'adapter à des besoins nouveaux; qu'il appartient néanmoins à l'autorité judiciaire d'apprécier si ces altérations de l'œuvre architecturale sont légitimées eu égard à leur nature et à leur importance, par les circonstances qui ont contraint le propriétaire à y procéder ${ }^{33}$. Les directives de proportionnalité aux besoins invoqués laissent à la vérité une bonne marge de manœuvre au juge ce que montrent l'affaire de la Maison Girard ${ }^{34}$ et plus récemment l'affaire de l'extension du musée d'Arles jugée par la Cour de cassation ${ }^{35}$. Soucieux de ce que, "pour préserver l'équilibre entre les prérogatives de l'auteur et celles du propriétaire de l'œuvre architecturale, les modifications apportées n'excèdent pas ce qui est strictement nécessaire à l'adaptation de l'œuvre à des besoins nouveaux et ne soient pas disproportionnées au regard du but poursuivi », le juge ici approuve la cour d'appel pour avoir décidé que «la découverte de la barque datant de l'époque romaine déclarée " trésor national » ainsi que de sa cargaison, et la nécessité d'exposer cet ensemble dans le musée considéré, caractérisent l'existence d'un besoin nouveau qui, pour être satisfait, commandait la construction d'une extension [...] et qu'il n'est pas démontré qu'elle dénature l'harmonie de l'œuvre ». L'architecture ici bridée dans son aspiration à la permanence. Voilà qui nous conduit à un autre partage d'utilités, dans lequel se joue le rapport de l'architecture à l'espace public. «La réalisation d'un architecte est rarement un morceau isolé, une phrase solitaire : en règle générale, elle résulte de l'échange avec les édifices environnants, avec les espaces urbains, avec les panoramas de la nature $»^{36}$. «L'architecture s'incarne nécessairement dans une époque et dans des lieux $\aleph^{37}$. Parce que l'œuvre est dans cet environnement, il est en prise avec 
d'autres intérêts publics, celui de la ville, protégée par le droit de l'urbain ou le droit du patrimoine, ceux du public et de ses droits d'accéder à l'œuvre. La loi a récemment introduit une exception de panorama, cependant timidement, contrairement à d'autres droits. Et c'est bien son implantation pérenne dans l'espace public, qui impose la franchise. Il y aurait sans doute à creuser davantage le rapport qu'entretient l'architecture au passé dans cet espace urbain, en particulier les liaisons qu'elle opère entre passé et présent.

\section{NOTES}

1. Sur leur gémellité, Pierre Caye, qui y voit deux savoirs de la différence et dans ce rapport à l'espace et deux arts de la distance, "Droit et Architecture. Savoirs de la différence, arts de la distance ", Académie des Sciences morales et politiques, séance du lundi 7 janvier 2008.

2. Robert Carvais, Dirk Van de Vijver, "Architecture et droit: mimésis théoriques et liaisons pratiques» dans Repenser les limites: l'architecture à travers l'espace, le temps et les disciplines, 31 août-4 septembre 2005.

3. Ibid.

4. Vincen Cornu, «Qu'est-ce que l'architecture? ", conférence donnée le 7 octobre 2009 à la Maison de l'architecture.

5. Décret sur la propriété des ouvrages par la voie de la presse ou de la gravure du 19 juillet 1793.

6. Rapport et décret sur la reconnaissance de la propriété intellectuelle, 19 juillet 1793, La Gazette nationale, 21 juillet $1793, \mathrm{n}^{\circ} 202$. Séance de la Convention nationale du 19 juillet 1793 , cité dans Philippe Poirrier, Les politiques culturelles en France, La documentation française, Comité d'histoire du ministère de la culture, CNFPT, Paris, 2002, p. 31.

7. Lakanal voit dans ce texte inaugural une « déclaration des droits du génie ».

8. À propos des arts industriels, Eugène Pouillet, Traité de la propriété littéraire et artistique et du droit de représentation, Imprimerie et Librairie générale de Jurisprudence, Marchal et Billard, $2^{\text {e }}$ édition 1894 , p. 89.

9. Même si dans certaines affaires, on discute d'éléments de décorations architecturaux, à propos d'un modèle de main pour marteau de porte protégé selon le juge comme toute œuvre des beauxarts, ibid., p. 93

10. Ibid., p. 108. Il a un développement spécial sur les œuvres d'architecture dans son traité. Dans le traité d'Henri Desbois, grand spécialiste de propriété intellectuelle, on trouve aussi un titre réservé aux œuvres d'architecture, Le droit d'auteur en France, $2^{\mathrm{e}}$ édition, Dalloz, Paris, 1967, p. 20.

11. Elle ne parle pas non plus des sculpteurs dont on admet pourtant sans discussion qu'ils sont des auteurs, sous réserve des controverses autour des arts industriels.

12. E. Pouillet, op. cit., p. 110.

13. Jean-Michel Leniaud, Les bâtisseurs d'avenir, portraits d'architectes, $X I X^{e}-X X^{e}$ siècle, Fayard, 1998, p. 134.

14. Jean-Michel Leniaud, « Noir sur Blanc », Livraisons d'histoire de l'architecture, $\mathrm{n}^{\circ}$ 30, 2015, p. 3.

15. Sur l'émergence d'un concepteur unique qui émergerait de la masse indistincte des constructeurs, Philippe Bernardi, «Quelques éléments de réflexion sur l'élaboration collective des projets architecturaux médiévaux », Livraisons d'histoire de l'architecture, $n^{\circ}$ 41, 2021, p. 2. 
16. Cour d'appel Paris, $1^{\mathrm{e}}$ chambre, 5 juin 1855, é, 28., cité dans Aurélie Chapon, « La protection de l'œuvre architecturale par le droit d'auteur ", Droit et Ville, 2013/2 (Nº 76), p. 11.

17. Sur ces débats autour de la propriété qui divise la profession, Jean-Michel Leniaud, Les bâtisseurs d'avenir, op. cit. p. 83.

18. Henri Desbois, op. cit. p. 20.

19. Patrizia Laudati. «Formes de l'architecture : langages, images et pratiques partagés » dans Pascal Lardellier. Formes en devenir. Approches technologiques, communicationnelles et symboliques, Hermes Science Publishing, 2014, Collection Science Cognitive et Management des Connaissances.

20. Agnès Callu, «La machine optique de l'architecture: desseins et dessins de Jean-Paul Jungmann, Livraisons d'histoire de l'architecture, $\mathrm{n}^{\circ} 30,2015$, p. 74

21. Jean-Michel Leniaud, « Noir sur blanc », Livraisons d'histoire de l'architecture, $\mathrm{n}^{\circ}$ 30, 2015, p. 3.

22. Sur cette notion de projet «qui concerne la conception en tant que telle, le moment où l'architecte projette, imagine ce qu'il va faire et le traduit par une production de dessins, de maquettes ", Bérénice Gaussuin, "Restauration des monuments historiques versus production nouvelle : l'encyclopédie d'architecture et la gazette des architectes et du bâtiment (1851-1869)», Livraison d'histoire de l'architecture, $\mathrm{n}^{\circ} 33,2017$, « Histoire du (des) patrimoine (s) », p. 24. L'auteur met en relation les mots de la restauration et ceux de la construction nouvelle.

23. Elle est «souvent le fait de sculpteurs, d'inventeurs ou d'artistes", Stéphanie QuantinBiancalani, «Pour une historiographie de la maquette d'architecture (XIX ${ }^{\mathrm{e}}-\mathrm{XX}^{\mathrm{e}}$ siècles) », Livraisons d'histoire de l'architecture $\mathrm{n}^{\circ}$ 39, p. 23.

24. Henri Desbois, op. cit., $\mathrm{n}^{\circ} 236$.

25. Vincen Cornu, op. cit.

26. Conseil d'État, $n^{\circ}$ 78833, 6 mai 1988, Dalloz, 1989, somm. 22, à propos de la rénovation et du réaménagement du bâtiment.

27. Encore que le concept juridique d'originalité se distingue du sens commun. Il n'est pas la nouveauté. Donné comme l'empreinte de la personnalité de l'auteur, diversement traduit aujourd'hui, l'effort, l'apport, etc., le critère sensé contenir l'enveloppe des créations juridiquement protégées n'est pas d'une grande efficience, paradoxalement.

28. Cour d'appel de Versailles, $1^{\mathrm{e}}$ chambre, 4 avril 1996 ; Facebat c/Sirvin, Semaine juridique, 11 décembre $1996, \mathrm{n}^{\circ} 22741$.

29. Nous laissons ce débat de côté, il faudrait rentrer plus finement dans les modes de structuration du collectif en architecture et la façon dont le droit, très imparfaitement les saisit.

30. L'œuvre de collaboration, véritable propriété commune mais en dépit de sa dénomination, l'œuvre collective n'en est pas une.

31. Philippe Bernardi, citant Franco Borsi, «Quelques éléments de réflexion sur l'élaboration collective des projets architecturaux médiévaux », op. cit., p. 2.

32. Cour de Cassation, $1^{\mathrm{e}}$ Chambre civile, du 7 janvier 1992, 90-17.534. Pour un exposé de cette histoire jurisprudentielle, et plus généralement sur les croisements droit et architecture, Michel Huet, Le droit de l'architecture, $3^{\mathrm{e}}$ édition, Économica, 2001 et L'architecte auteur: Pratiques quotidiennes du droit d'auteur en architecture, paysage et urbain, Le Moniteur, 2006.

33. La formule sur la non intangibilité de l'œuvre sera reprise dans de nombreuses affaires, pour beaucoup des réalisations publiques, stade, place publique, musée, médiathèque, etc., plaidées tantôt devant le juge judiciaire, tantôt devant le juge administratif, ce dernier se montrant parfois plus favorable à l'auteur.

34. Les juges vont justifier la destruction de l'édifice faisant peu de cas en l'espèce des droits de l'auteur.

35. Cour de cassation, civile, $1^{\mathrm{e}}$ Chambre civile, 20 décembre 2017, 16-13.632, inédit.

36. André Chastel, « Voir l'architecture, problèmes et besoins ", Architecture et Patrimoine, choix de chroniques parues dans le Monde, Éditions du patrimoine, Centre des monuments nationaux, 
$2^{\mathrm{e}}$ édition, Paris, 2012, p. 124 citant un ouvrage de B. Zevi, Saper vedere l'archittetura, Turin 1948, $5^{\mathrm{e}}$ éd. 1956.

37. Vincen Cornu, «Qu'est-ce que l'architecture ?», op. cit.

\section{RÉSUMÉS}

Le thème de l'architecture comme processus de création, présent dans de nombreuses Livraisons d'histoire de l'architecture, ne manque pas de poser la question des rapports qu'entretiennent le droit et l'architecture, celle des discours que tient chacun sur l'art de construire. Quels sont les espaces de dialogues entre ces deux sciences? Que peut nous enseigner ce côtoiement? Toutes deux affrontent la figure de l'architecte auteur, interrogent les multiples formes qu'il produit. Fruit de l'art et de la technique, l'architecture est un lieu de partage d'utilités privées comme publiques. C'est ce qui fait que, dans le champ de la création, l'œuvre architecturale est singulière.

The theme of architecture as a creative process, present in numerous Livraisons d'histoire de l'architecture, does not fail to raise the question of the relationship between law and architecture, and the discourse that each holds on the art of building. What are the spaces of dialogue between these two sciences? What can we learn from this interaction? Both confront the figure of the architect as author, questioning the multiple forms that he produces. Fruit of the art and the technique, the architecture is a place of sharing of private as public utilities. This is what makes the architectural work singular in the field of creation.

Das Thema der Architektur als kreativer Prozess, das in vielen Livraisons d'histoire de l'architecture vorkommt, wirft die Frage nach der Beziehung zwischen Recht und Architektur und dem Diskurs auf, den beide über die Kunst des Bauens führen. Welche Räume gibt es für den Dialog zwischen diesen beiden Wissenschaften? Was kann uns diese Nähe lehren? Beide setzen sich mit der Figur des Architekten als Autor auseinander und hinterfragen die vielfältigen Formen, die er produziert. Als Ergebnis von Kunst und Technik ist die Architektur ein Ort, an dem private und öffentliche Nutzungen geteilt werden. Das macht das architektonische Werk einzigartig auf dem Gebiet der Schöpfung.

\section{AUTEUR}

\section{MARIE CORNU}

Marie Cornu est directrice de recherches au CNRS affectée à l'ISP (Institut des Sciences sociales du politique, UMR 7220, ENS Paris Saclay, Université Paris X Nanterre). Ses thématiques portent en grande partie sur le droit du patrimoine ainsi que sur les rapports entre langue et droit et plus généralement sur l'évolution des figures de la propriété. Elle a publié : Le droit culturel des biens, l'intérêt culturel juridiquement protégé, Bruylant, 1996 et co-dirigé plusieurs ouvrages collectifs notamment le Dictionnaire de droit comparé du droit du patrimoine culturel, co-auteurs Jérôme Fromageau, Catherine Wallaert, CNRS, 2012, le Dictionnaire des biens communs, PUF, 2 ème édition, 2021 (dir. M. Cornu, F. Orsi, J. Rochfeld), et dans le cadre du projet Mémoloi, 1913, genèse d'une loi sur les monuments historiques, 2013 et De 1913 au Code du patrimoine, une loi en évolution, 2018, (dir. J.- 
P. Bady, M. Cornu, J. Fromageau, J.-M. Leniaud, V. Négri), 1979, genèse d'une loi (dir. M. Cornu, Y. Potin, B. Ricard, N. Wagener), Comité d'histoire du ministère de la Culture, documentation française, « La mort des cathédrales, Marcel Proust et la séparation des Églises et de l'État », Revue Droit \& Littérature, 2021/1 ( ${ }^{\circ}$ 5), p. 177-194. 